\title{
A macroscopic traffic model for traffic flow harmonization
}

\author{
Zawar H. Khan ${ }^{1 *}$ and T. Aaron Gulliver ${ }^{2}$
}

\begin{abstract}
Traffic flow will harmonize to forward conditions. The time and distance required for harmonization can have a significant effect on the traffic density behavior. The flow can evolve into clusters of vehicles or become uniform depending on parameters such as safe time headway and safe distance headway. In this paper, a new model is presented to provide a realistic characterization of traffic behavior during the harmonization period. Results are presented for a discontinuous density distribution on a circular road which shows that this model produces more realistic traffic behavior than other models in the literature.
\end{abstract}

Keywords: Macroscopic traffic model, Reaction distance, Reaction time, Safe distance headway, Safe time headway, Traffic flow, Roe decomposition

\section{Introduction}

This paper considers the behavior of vehicles as they harmonize to forward traffic conditions. The time for traffic harmonization is based on the front traffic stimuli, i.e. the time to react and align (harmonize) to the forward traffic. The time required to react is known as the reaction time, and the time for traffic alignment (harmonization) is known as the transition time [1] The reaction distance is the distance travelled during the reaction time, while the transition distance is the distance covered during the transition time. The sum of the transition and reaction times is known as the safe time headway. This is the time required for the safe adjustment of velocity. The distance travelled during the safe time is known as the safe distance headway.

Drivers adjust their velocity when a change in traffic flow is observed in an effort to achieve the equilibrium velocity distribution. This distribution depends on the traffic density as well as driver behavior and road characteristics, and will result in a homogeneous traffic flow [2]. This flow will evolve into clusters with a large safe distance headway and small safe time headway. Conversely, a small safe distance headway and large safe time headway will produce a more uniform flow. The goal of this paper is to develop a simple,

\footnotetext{
* Correspondence: zawarkhan@nwfpuet.edu.pk

${ }^{1}$ Electrical Engineering Department, University of Engineering and

Technology, Peshawar, Pakistan

Full list of author information is available at the end of the article
}

realistic model to characterize the traffic flow. This will lead to better control of traffic behavior to mitigate congestion, reduce pollution levels, and improve public safety. These models can also be employed for automatic control of traffic flow to reduce travel time.

The main types of traffic models are macroscopic, mesoscopic and microscopic. Macroscopic models consider the aggregate behavior of traffic flow while microscopic models consider the interaction of individual vehicles. These models include parameters such as driver behavior, vehicle locations, distance headways, time headways, and the velocity and acceleration of individual vehicles. Mesoscopic models share the properties of macroscopic and microscopic traffic models. These models characterize the influence of vehicles in close proximity and then approximate the cumulative temporal and spatial traffic behavior [3]. Macroscopic traffic models are typically employed due to their low complexity.

Newel proposed a microscopic traffic model and acknowledged for the first time that the distance headways varies during traffic harmonization [4], but the variable distances between vehicles were not explained [5]. The General Motors model indicates that the distance headway between vehicles increases with velocity. However, this model ignores the variable distances between vehicles at slow speeds [6, 7]. Gipps characterized variable distance headways using a safety rule [8]. According to this model, the distance headways 
between vehicles should be sufficient to decelerate and harmonize speeds. However, the behavior of the driver population on the distance headway was not considered. Wiedemann proposed a psychophysical model to characterize individual driver behavior based on conscious and unconscious reaction and perception [9]. Drivers unconsciously harmonize small changes in speed for small distance headways, so the reaction of these drivers is slow. Conversely, with large distance headways, drivers consciously harmonize speeds with large reactions and perceptions. The limitations of the Wiedemann model are the constant ranges for perception and reaction which actually vary depending on the speed harmonization [10].

Driver behavior varies with ethnicity, gender, age, psychology and intoxication level. People in the age groups 18-25, 25-55 and 55+ have different cognitive and physical behavior and thus also different driving behavior [11]. Cognitive and physical behavior decline with age which increases the probability of accidents. The crash rate between ages 35-64 is three times less than at $65+$ [12]. Older people can have difficulties moving their heads sideways to scan the traffic flow and there can be memory issues. Thus, reaction to changing conditions can be sluggish and is a major cause of accidents at intersections [11, 13]. Young drivers often exhibit limited horizontal road scanning behavior which is similar to the sluggish behavior of intoxicated drivers [14]. Drugs and alcohol change thought processes and typically lead to slow driving behavior. In 1972, teenagers in the US were five times more likely to die in a traffic accident than people in the 35-64 age group. Experienced drivers have a wide horizontal traffic scanning behavior. Thus, they quickly recognize changes in speed and density and so take less time to perceive and react than inexperienced drivers. Existing microscopic models do not characterize differences in driving behavior. To more accurately characterize traffic flow harmonization (alignment), a model is required which captures the effect of driver behavior [5]. This behavior can be characterized for specific groups such as intoxicated drivers, old and young drivers, and considering ethnicity and experience.

One of the most popular macroscopic traffic flow models is the two equation model developed by Payne [15] and Whitham [16] which is known as the PayneWhitham (PW) model. The first equation is based on the continuity equation for the conservation of vehicles on a road, while the second models the acceleration behavior of traffic based on driver anticipation and relaxation. Driver anticipation results from the presumption of changes in the forward traffic density, while relaxation is the tendency to adjust velocity based on traffic conditions. The relaxation time can be considered the transition time. The PW model is based on the assumption that vehicles on a road have similar behavior. Smooth traffic velocities and density distributions are employed [17], and alignment (harmonization) occurs with a constant velocity [18]. Unfortunately, this results in unrealistic velocity and density behavior [19]. Zhang improved the PW model using the fact that driver anticipation cannot be constant [20], and considered that drivers harmonize their speeds based on the density distribution. However, this model can produce unrealistic results in some traffic flow situations.

Khan and Gulliver [21] improved the PW model using the fact that driver anticipation is based on the velocity of the forward traffic, so that traffic behavior depends on the velocity during transitions. It was shown that this model provides more realistic traffic flow and density than the PW model. The sensitivity of traffic is the rate at which alignment occurs. The limitation of the KhanGulliver (KG) model is that this sensitivity depends only on the relaxation time $\tau$. For high velocities, $\tau$ is small, so traffic alignment can occur too quickly, whereas with low velocities, $\tau$ is large so alignment can be very slow. As a consequence, this model does not have sufficient flexibility to properly characterize traffic behavior.

In this paper, a new model is proposed to provide more realistic traffic behavior ranging from vehicle clusters to a uniform flow. Transitions in the flow occur when vehicles enter or leave at connecting roads, or when there are obstructions or bottlenecks on the road. The resulting harmonization is affected by the flow behavior and safe velocity given by

$$
v_{s}=\frac{d_{s}}{t_{s}}
$$

where $d_{s}$ is the safe distance headway and $t_{s}$ is the safe time headway. The traffic density distribution has a greater variance at lower safe velocities [21], and changes in this distribution during alignment depend on the velocity adjustments required to adapt to the equilibrium velocity distribution. In the proposed model, a parameter is introduced to regulate traffic flow behavior so these adjustments are appropriate. With a large regulation value, the flow evolves into a large number of small clusters. Conversely, the traffic flow is more uniform with a small value. This value can be extended to incorporate driving behavior due to factors such as intoxication, experience, ethnicity, and age, to accurately model traffic behavior. It can also be employed for traffic during adverse weather and congestion, and movement in clusters. The effect of this parameter is examined in Section IV.

The remainder of this paper is organized as follows. Section II presents the KG, Zhang and proposed models. In Section III, the well-known Roe decomposition technique is used to implement these models, and performance 
results are presented for a circular road in Section IV. Finally, some concluding remarks are given in Section V.

\section{Traffic flow models}

The KG model [21] was developed to characterize traffic flow behavior according to forward velocity conditions. The KG model in conservation form is given by

$$
\begin{aligned}
& \rho_{t}+(\rho v)_{x}=0 \\
& (\rho v)_{t}+\left(\frac{(\rho v)^{2}}{\rho}+\left(\frac{v^{2}(\rho)-v^{2}}{2 d_{t r}}\right) \rho\right)_{x}=\rho\left(\frac{v(\rho)-v}{\tau}\right),
\end{aligned}
$$

where the subscripts $t$ and $x$ denote temporal and spatial derivatives, respectively. $\rho$ and $v$ are the traffic density and average velocity, respectively, so that $\rho v$ is the flow. $v(\rho)$ is the equilibrium velocity distribution and $d_{t r}$ is the transition distance. A large average velocity results in a small relaxation time and thus the alignment (harmonization) can be too fast and produce unrealistic behavior.

The source term in (3) is

$$
\rho\left(\frac{v(\rho)-v}{\tau}\right)
$$

which indicates that traffic alignment (harmonization) occurs according to the difference between the average velocity and the equilibrium velocity distribution. In reality, alignment is faster at higher velocities, so it should not be a function of only this difference. After harmonization, the source term is zero, i.e. $v=v(\rho)$, and the traffic flow is smooth. The sensitivity of this term is given by

$$
\zeta_{1}=\frac{1}{\tau}
$$

and this determines how quickly harmonization occurs given the other parameters in (4). Thus, it can have a significant effect on traffic behavior. However, $\zeta_{1}$ only depends on the relaxation time $\tau$, which may not be sufficient to produce appropriate traffic behavior.

The following traffic model based on the forward traffic stimuli was presented in [21]

$$
\rho_{t}+\left(\rho\left(\frac{v(\rho)^{2}-v^{2}}{2 v_{s}}\right)\right)_{x}=0 .
$$

This model has been used to characterize traffic behavior during transitions as well as when the flow is smooth. The right-hand side (RHS) of (6) is zero because the traffic is considered to be on a long infinite road with no transitions due to the ingress or egress of vehicles to the flow. The anticipation term of this model

$$
\rho\left(\frac{v^{2}(\rho)-v^{2}}{2 v_{s}}\right)
$$

characterizes the driver presumption of changes in the forward traffic. With this model, traffic alignment (harmonization) is a quadratic function of velocity. Further, the sensitivity of (7) is

$$
\zeta_{2}=\frac{1}{2 v_{s}}
$$

so it is also a function of the safe distance headway and safe time headway, and alignment occurs according to the inverse of the safe velocity.

In this paper, a new model is proposed for transition harmonization in a traffic flow by characterizing the driver response using (7). If the equilibrium velocity $v(\rho)$ is greater than the average velocity $v$ there is acceleration and alignment will occur at a velocity greater than $v$. Conversely, if $v(\rho)$ is smaller than $v$ there is deceleration, and alignment will occur at a velocity smaller than $v$. This can be characterized by the numerator of (7). Further, alignment (harmonization) depends on the physiological and psychological response of the drivers. This behavior can be characterized by modifying the denominator of (7). Thus, the number 2 in (7) is replaced with a flow regulation value $b$. A small value of $b$ will produce a more uniform flow, while a large value will result in clustered traffic. The new source term is then

$$
\rho\left(\frac{v^{2}(\rho)-v^{2}}{b \frac{d_{s}}{t_{s}}}\right) .
$$

The safe distance headway consists of the reaction distance $d_{r}$ and transition distance $d_{t r}$ so that

$$
\zeta_{3}=\frac{1}{b \frac{d_{s}}{t_{s}}} .
$$

The psychological response of a driver is characterized by the transition distance, and the physiological response by the reaction distance. A sluggish driver responds slowly and takes more time to perceive and process forward traffic conditions. Thus, they will have large reaction and transition distances to align to the traffic. Examples of sluggish drivers are intoxicated or distracted drivers, old and new drivers, and drivers affected by fatigue [22, 23]. The reaction time in a fatigued state has been shown to be $17 \%$ longer than in an alert state, and the increase in reaction time is greater for females than males [24]. Further, old drivers have longer reaction times than young drivers. For a sluggish driver, $b$ should be large. Conversely, excited or aggressive drivers such as commercial or teenage drivers 
will have a small reaction time and corresponding small transition distance [18], so $b$ should be small.

Replacing the source term in (3) of the KG model with (9) gives

$$
(\rho v)_{t}+\left(\frac{(\rho v)^{2}}{\rho}+\left(\frac{v^{2}(\rho)-v^{2}}{2 d_{t r}}\right) \rho\right)_{x}=\rho\left(\frac{v^{2}(\rho)-v^{2}}{b v_{s}}\right) .
$$

for the new model, while (2) is not changed. The PW model is given by $[15,16,21]$

$$
\begin{aligned}
\rho_{t}+(\rho v)_{x} & =0 \\
\rho v_{t}+\rho v v_{x}+\rho C_{0}^{2} \rho_{x} & =\rho \frac{v(\rho)-v}{\tau},
\end{aligned}
$$

where $C_{0}$ is the velocity constant which characterizes driver response. According to this model, driver response does not depend on the traffic conditions and is a constant. The relaxation term of the PW model is the same as that in the KG model, as shown in Table 1. Several models have been proposed for $v(\rho)$ [25], but the most commonly employed is the Greenshields model which is given by [26]

$$
v(\rho)=v_{m}\left(1-\frac{\rho}{\rho_{m}}\right)
$$

where $\rho_{m}$ and $\rho$ are the maximum and average traffic densities, respectively, and $v_{m}$ is the maximum velocity on the road. Therefore, this model is employed here.

The Zhang model is given by [20]

$$
\begin{gathered}
\rho_{t}+(\rho v)_{x}=0 \\
\rho v_{t}+\rho v v_{x}+\frac{\left(\rho \frac{\partial v(\rho)}{\partial \rho}\right)^{2}}{\rho} \rho_{x}=\rho \frac{v(\rho)-v}{\tau}
\end{gathered}
$$

The relaxation term is the same as the PW and KG models, as shown in Table 1. With this model, the driver response depends on the traffic density. The next section presents Roe's decomposition technique which is used to implement the KG, PW, Zhang, and proposed models.

\section{Roe decomposition}

The KG, PW, Zhang and proposed models are discretized using the decomposition technique developed by Roe [27] to evaluate their performance. This technique can be used to approximate the nonlinear system of equations

$$
G_{t}+f(G)_{x}=S(G),
$$

where $G$ is the vector of data variables, $f(G)$ is the vector of functions of these variables, and $S(G)$ is the vector of source terms. The subscripts $t$ and $x$ denote partial derivatives with respect to time and distance, respectively. Equation (14) is then given by

$$
\frac{\partial G}{\partial t}+\frac{\partial f}{\partial G} \frac{\partial G}{\partial x}=S(G)
$$

Let $A(G)$ be the Jacobian matrix of the system. Then (15) can be expressed as

$$
\frac{\partial G}{\partial t}+A(G) \frac{\partial G}{\partial x}=S(G) .
$$

Setting the source terms in (16) to zero gives the quasilinear form

$$
\frac{\partial G}{\partial t}+A(G) \frac{\partial G}{\partial x}=0
$$

The data variables are density $\rho$ and flow $\rho v$ in the KG, PW, Zhang and proposed models. Roe's technique is used to linearize the Jacobian matrix $A(G)$ by decomposing it into eigenvalues and eigenvectors. This is based on the realistic assumption that the data variables, eigenvalues and eigenvectors remain conserved for small changes in time and distance. This technique is widely employed because it is able to capture the effects of abrupt changes in the data variables.

Consider a road divided into $M$ equidistant segments and $N$ equal duration time steps. The total length is $x_{M}$ so a road segment has length $\delta x=x_{M} / M$, and the total time period is $t_{N}$ so a time step is $\delta t=t_{N} / N$. The Jacobian matrix is approximated for road segments $\left(x_{i}+\frac{\delta x}{2}, x_{i}-\frac{\delta x}{2}\right)$. This matrix is determined for all $M$ segments in every time interval $\left(t_{n+1}, t_{n}\right)$, where $t_{n+1}-t_{n}=\delta t$.

Let $\Delta G$ be a small change in the data variables $G$ and $\Delta f$ the corresponding change in the functions of these variables. Further, let $G_{i}$ be the average value of the data

\begin{tabular}{|c|c|c|c|c|}
\hline Term & KG model & PW model & Proposed model & Zhang model \\
\hline Anticipation & $\rho \frac{\partial}{\partial x}\left(\frac{u^{2}(\rho)-v^{2}}{2 d_{t r}}\right) \rho_{x}$ & $\rho C_{0}^{2} \rho_{x}$ & $\rho \frac{\partial}{\partial x}\left(\frac{u^{2}(\rho)-u^{2}}{2 d_{t r}}\right) \rho_{x}$ & $\frac{\left(\frac{\partial(\rho) \rho}{\partial \rho}\right)^{2}}{\rho} \rho_{x}$ \\
\hline Relaxation & $\rho \frac{U(\rho)-U}{\tau}$ & $\rho \frac{U(\rho)-U}{\tau}$ & $\rho\left(\frac{U^{2}(\rho)-u^{2}}{b u_{s}}\right)$ & $\rho \frac{U(\rho)-U}{\tau}$ \\
\hline
\end{tabular}
variables in the $i$ th segment. The change in flux at the boundary between the $i$ th and $(i+1)$ th segments is

$$
\Delta f_{i+\frac{1}{2}}=A\left(G_{i+\frac{1}{2}}\right) \Delta G,
$$

where $A\left(G_{i+\frac{1}{2}}\right)$ is the Jacobian matrix at the segment boundary, and $G_{i+\frac{1}{2}}$ is the vector of data variables at the boundary obtained using Roe's technique. The flux

Table 1 Traffic model comparison 
approximates the change in traffic density and flow at the segment boundary. We have that $A\left(G_{i+\frac{1}{2}}\right)=e \Lambda e^{-1}$, where $\Lambda$ is a diagonal matrix of the eigenvalues $\left[\lambda_{1}, \lambda_{2}\right.$, $\cdots, \lambda_{p}$ ] of the Jacobian matrix and $e$ is the corresponding eigenvector matrix. From [28], the eigenvalues should be positive so that

$$
\Delta f_{i+\frac{1}{2}}=e|\Lambda| e^{-1}\left(G_{i+1}-G_{i}\right),
$$

where the approximation $\Delta G=\left(G_{i+1}-G_{i}\right)$ is used. The flux at the boundary between segments $i$ and $i+1$ at time $n$ is then approximated by

$$
f_{i+\frac{1}{2}}^{n}\left(G_{i}^{n}, G_{i+1}^{n}\right)=\frac{1}{2}\left(f\left(G_{i}^{n}\right)+f\left(G_{i+1}^{n}\right)\right)-\frac{1}{2} \Delta f_{i+\frac{1}{2}},
$$

where $f\left(G_{i}^{n}\right)$ and $f\left(G_{i+1}^{n}\right)$ denote the values of the functions of the data variables in road segments $i$ and $i+1$ respectively, at time $n$. Substituting (19) into (20) gives

$$
\begin{aligned}
f_{i+\frac{1}{2}}^{n}\left(G_{i}^{n}, G_{i+1}^{n}\right)= & \frac{1}{2}\left(f\left(G_{i}^{n}\right)+f\left(G_{i+1}^{n}\right)\right) \\
& -\frac{1}{2} e|\Lambda| e^{-1}\left(G_{i+1}^{n}-G_{i}^{n}\right) .
\end{aligned}
$$

This approximates the change in density and flow without considering the source.

For the source decomposition of the KG model in (3), the PW model $[15,20]$ in (12) and the Zhang model in (14), we have

$$
S_{1}\left(G_{i}^{n}\right)=\rho_{i}^{n}\left(\frac{v\left(\rho_{i}^{n}\right)-v_{i}^{n}}{\tau}\right)
$$

and for the proposed model in (11)

$$
S_{2}\left(G_{i}^{n}\right)=\rho_{i}^{n}\left(\frac{v^{2}\left(\rho_{i}^{n}\right)-\left(v_{i}^{n}\right)^{2}}{b v_{s}}\right) .
$$

The updated data variables for the KG, PW, Zhang and proposed models are

$$
G_{i}^{n+1}=G_{i}^{n}-\frac{\delta t}{\delta x}\left(f_{i+\frac{\delta}{2}}^{n}-f_{i+\frac{\delta x}{2}}^{n}\right)+\delta t S_{y}\left(G_{i}^{n}\right), y=1,2 .
$$

Both the KG and proposed models have the same expressions on the left-hand side (LHS) as shown in (3) and (11). Therefore, the Jacobian matrix $A(G)$ is the same for these models and results in the same eigenvalues and eigenvectors, and also average velocity and density. $A(G)$ as well as the corresponding eigenvalues and eigenvectors, average density and velocity for the KG model were derived in [21]. Assuming the right hand side (RHS) of the KG and proposed models are in quasilinear form, traffic flow alignment (harmonization) is not considered so that $S_{y}(G)=\left(\frac{0}{0}\right)$. Then (24) takes the form

$$
G=\left(\frac{\rho}{\rho v}\right), f(G)=\left(\frac{f_{1}}{f_{2}}\right)=\left(\frac{\rho v}{\frac{(\rho v)^{2}}{\rho}+\frac{v^{2}(\rho)-v^{2}}{2 d_{t r}} \rho}\right) \text { and } S_{y}(G)=\left(\frac{0}{0}\right) \text {. }
$$

The LHS of (3) and (11) is approximated using the Jacobian matrix $(G)=\frac{\partial f}{\partial G}$, which is obtained from (25).

The Jacobian matrix $A(G)=\frac{\partial f}{\partial G}$ from (25) is

$$
A(G)=\left(\frac{0}{-v^{2}+\left(\frac{v^{2}(\rho)-v^{2}}{2 d_{t r}}\right)} \frac{1}{2 v}\right)
$$

The eigenvalues obtained from (26) in Appendix A are

$$
\lambda_{1,2}=v \pm \sqrt{\left(\frac{v^{2}(\rho)-v^{2}}{2 d_{t r}}\right)} .
$$

These show that when a transition occurs, the velocity changes according to the equilibrium velocity distribution and the average velocity.

For a traffic flow to be strictly hyperbolic, the eigenvectors must be distinct and real [29]. The eigenvectors corresponding to the eigenvalues in (27) are distinct and real when the equilibrium velocity is greater than the average velocity, i.e.

$$
v\left(\rho_{i+\frac{1}{2}}\right)>v_{i+\frac{1}{2}}
$$

Conversely, the eigenvectors are imaginary when

$$
v\left(\rho_{i+\frac{1}{2}}\right)<v_{i+\frac{1}{2}}
$$

so to maintain the hyperbolic property for the proposed model, the absolute value of the numerator under the radical sign in (27) is employed, which gives

$$
\lambda_{1,2}=v_{i+\frac{1}{2}} \pm \sqrt{\frac{\left|v^{2}\left(\rho_{i+\frac{1}{2}}\right)-v_{i+\frac{1}{2}}^{2}\right|}{2 d_{t r}}} .
$$

The corresponding eigenvectors of the KG and proposed models are 


$$
e_{1,2}=\left(\frac{1}{v_{i+\frac{1}{2}} \pm \sqrt{\frac{\left|v^{2}\left(\rho_{i+\frac{1}{2}}\right)-v_{i+\frac{1}{2}}^{2}\right|}{2 d_{t r}}}}\right) .
$$

The eigenvalues and eigenvectors, average density and velocity for the PW model were derived in [18]. The eigenvalues are

$$
\lambda_{1,2}=v_{i+\frac{1}{2}} \pm C_{0},
$$

where $C_{0}$ is the velocity constant. This shows that traffic velocity alignment is at a constant rate $C_{0}$ during transitions. The corresponding eigenvectors are

$$
e_{1,2}=\left(\frac{1}{v_{i+\frac{1}{2}} \pm C_{0}}\right)
$$

The average velocity at the boundary of segments $i$ and $i+1$ for the KG, PW and proposed models is

$$
v_{i+\frac{1}{2}}=\frac{v_{i+1} \sqrt{\rho_{i}+1}+v_{i} \sqrt{\rho_{i}}}{\sqrt{\rho_{i}+1}+\sqrt{\rho_{i}}}
$$

The average density for these models at the boundary of segments $i$ and $i+1$ is given by the geometric mean of the densities in these segments

$$
\rho_{i+\frac{1}{2}}=\sqrt{\rho_{i+1} \rho_{i}} .
$$

The eigenvalues for the Zhang model [25] are

$$
\lambda_{1}=v_{i+\frac{1}{2}},
$$

and

$$
\lambda_{2}=v_{i+\frac{1}{2}}+\rho v(\rho)_{\rho}
$$

where the subscript $\rho$ presents the derivative of the equilibrium velocity distribution with respect to density. The corresponding eigenvectors are

$$
\begin{aligned}
& e_{1}=\left(\frac{1}{v_{i+\frac{1}{2}}-v\left(\rho_{i+\frac{1}{2}}\right)-\rho_{i+\frac{1}{2}} v\left(\rho_{i+\frac{1}{2}}\right)_{\rho}}\right), \\
& e_{2}=\left(\frac{1}{v_{i+\frac{1}{2}}-v\left(\rho_{i+\frac{1}{2}}\right)}\right) .
\end{aligned}
$$

The average density $\rho_{i+\frac{1}{2}}$ of the Zhang model at the segment boundary is the same as for the KG, PW and proposed models given in (33). The average velocity $v_{i+\frac{1}{2}}$ at the boundary of segments $i$ and $i+1$ for the Zhang model is given in Appendix B.

\section{A. Entropy Fix}

Entropy fix is applied to Roe's technique to smooth any discontinuities at the segment boundaries. The Jacobian matrix $A\left(G_{i+\frac{1}{2}}\right)$ is decomposed into its eigenvalues and eigenvectors to approximate the flux in the road segments (21). The Jacobian matrix for the road segments is then replaced with the entropy fix solution given by

$$
e|\Lambda| e^{-1},
$$

where $|\Lambda|=\left[\hat{\lambda}_{1}, \hat{\lambda}_{2}, \cdots, \hat{\lambda}_{k}, \cdots, \hat{\lambda}_{n}\right]$ is a diagonal matrix which is a function of the eigenvalues $\lambda_{k}$ of the Jacobian matrix, and $e$ is the corresponding eigenvector matrix. The Harten and Hayman entropy fix scheme [30] is employed here so that

$$
\hat{\lambda}_{k}=\left\{\begin{array}{llr}
\hat{\delta}_{k} \text { if } & \left|\lambda_{k}\right|<\hat{\delta}_{k} \\
\left|\lambda_{k}\right| & \text { if } & \left|\lambda_{k}\right| \geq \hat{\delta}_{k}
\end{array}\right.
$$

with

$$
\hat{\delta}_{k}=\max \left(0, \quad \lambda_{i+\frac{1}{2}}-\lambda_{i}, \quad \lambda_{i+1}-\lambda_{i+\frac{1}{2}}\right) .
$$

This ensures that the $\hat{\lambda}_{k}$ are not negative and similar at the segment boundaries. The Jacobian matrix $e|\Lambda| e^{-1}$ for the proposed, KG and Zhang models are given in Appendix $\mathrm{C}$. The corresponding flux is obtained from (21) using $f\left(G_{i}\right)$ and $f\left(G_{i+1}\right)$ and substituting $e|\Lambda| e^{-1}$ for $A\left(G_{i+\frac{1}{2}}\right)$ , the updated data variables, $\rho$ and $\rho v$, are then obtained at time $n$ using (24).

\section{Performance results}

The performance of the proposed model is evaluated in this section and compared with the KG, PW and Zhang models over a circular road of length $x_{M}=100 \mathrm{~m}$. A discontinuous density distribution $\rho_{0}$ at $t=0$ with periodic boundary conditions is employed. $\rho_{0}$ is shown in blue in the figures. The Greenshields equilibrium velocity distribution given in (13) is used with $v_{m}=34 \mathrm{~m} / \mathrm{s}$ and maximum density $\rho_{m}=1$. The safe distance headway is $28 \mathrm{~m}$, the safe time headway is $t_{s}=1.4 \mathrm{~s}$, and $d_{t r}$ is $20 \mathrm{~m}$. For the KG and PW models, $\tau=1 \mathrm{~s}$. The total simulation time for the proposed and KG models is $30 \mathrm{~s}$. The total simulation time for the PW model is $3 \mathrm{~s}$ and for the Zhang model is $4 \mathrm{~s}$. Based on $\delta x=1 \mathrm{~m}$, the time step for the proposed, KG and Zhang models is chosen as $\delta t=0.01 \mathrm{~s}$, and the time step for the PW model is chosen as $\delta t=0.006 \mathrm{~s}$, to satisfy the CFL condition [31]. The number of time steps and road steps are 3000 and 100, respectively, for both the proposed and KG models, while the number of time steps 
for the PW model is 500. The number of road steps and time steps for the Zhang model are 100 and 400, respectively. The flow regulation parameters considered for the proposed model are $b=1$ and 2 . The simulation parameters are summarized in Table 2.

Figure 1 presents the normalized traffic density with the KG model at four different time instants. This shows that the traffic evolves into two clusters of vehicles. At $5 \mathrm{~s}$ the density behavior is slightly oscillatory. However, at $15 \mathrm{~s}$ the traffic density beyond $50 \mathrm{~m}$ has an almost uniform level of 0.09 , and there are two clusters of vehicles between 0 and $50 \mathrm{~m}$. The traffic density of these clusters ranges from 0.1 to 0.21 . Both clusters span a distance of approximately $20 \mathrm{~m}$. At $30 \mathrm{~s}$, the traffic density between 0 and $40 \mathrm{~m}$ has an almost uniform density of 0.09 , while beyond $40 \mathrm{~m}$ there are two clusters. The clusters still span a distance of about $20 \mathrm{~m}$, so they have just moved over time. The first cluster has a maximum density of 0.25 at $50 \mathrm{~m}$, and the second a maximum density of 0.2 at $78 \mathrm{~m}$.

Table 2 Simulation parameters

\begin{tabular}{|c|c|c|}
\hline Name & Parameter & Value \\
\hline road step & $\delta x$ & $1 \mathrm{~m}$ \\
\hline equilibrium velocity & $u(\rho)$ & $\begin{array}{l}\text { Greenshields velocity } \\
\text { distribution }\end{array}$ \\
\hline maximum velocity & $u_{m}$ & $34 \mathrm{~m} / \mathrm{s}$ \\
\hline $\begin{array}{l}\text { time step for the KG, Zhang } \\
\text { and proposed models }\end{array}$ & $\delta t$ & $0.01 \mathrm{~s}$ \\
\hline time step for the PW model & $\delta t$ & $0.006 \mathrm{~s}$ \\
\hline velocity constant & $C_{0}$ & $4.12 \mathrm{~s}$ \\
\hline safe distance headway & $d_{s}$ & $28 \mathrm{~m}$ \\
\hline transition distance & $d_{t r}$ & $20 \mathrm{~m}$ \\
\hline safe time headway & $t_{s}$ & $1.4 \mathrm{~s}$ \\
\hline safe velocity & $u_{s}$ & $\frac{28}{1.4}=20 \mathrm{~m} / \mathrm{s}$ \\
\hline normalized maximum density & $\rho_{m}$ & 1 \\
\hline $\begin{array}{l}\text { total simulation time for the } \\
\mathrm{KG} \text { and proposed models }\end{array}$ & $t_{N}$ & $30 \mathrm{~s}$ \\
\hline $\begin{array}{l}\text { total simulation time for the } \\
\text { PW model }\end{array}$ & $t_{N}$ & $3 s$ \\
\hline $\begin{array}{l}\text { total simulation time for the } \\
\text { Zhang model }\end{array}$ & $t_{N}$ & $4 \mathrm{~s}$ \\
\hline flow regulation parameter & B & 1,2 \\
\hline relaxation time constant & $T$ & $1 \mathrm{~s}$ \\
\hline $\begin{array}{l}\text { number of time steps for the } \\
\text { PW model }\end{array}$ & N & 500 \\
\hline $\begin{array}{l}\text { number of time steps for the KG } \\
\text { and proposed models }\end{array}$ & N & 3000 \\
\hline $\begin{array}{l}\text { number of time steps for the } \\
\text { Zhang model }\end{array}$ & $N$ & 400 \\
\hline number of road steps & M & 100 \\
\hline
\end{tabular}

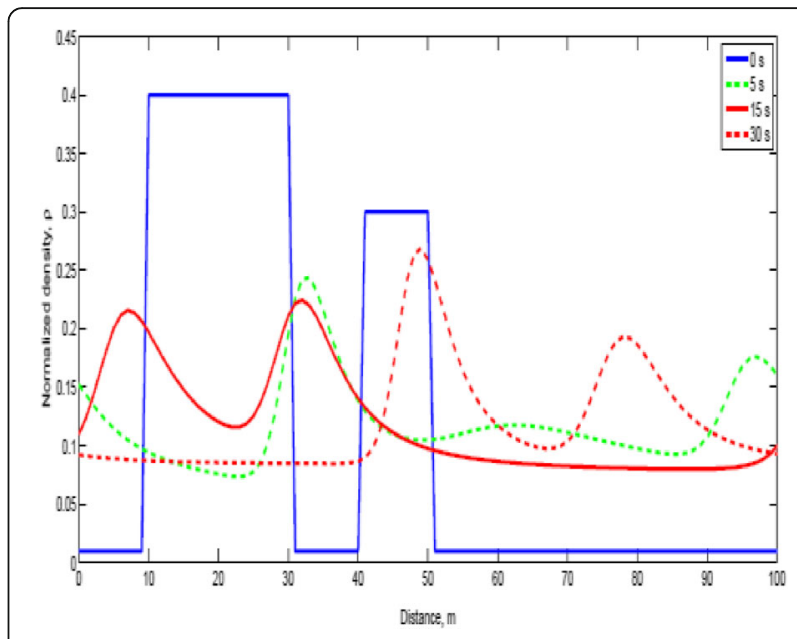

Fig. 1 The KG model density behavior with $\tau=1 \mathrm{~s}$

Figure 2 presents the normalized traffic density with the proposed model and $b=1 \mathrm{~s}$ at four different time instants. This shows that with a small value of $b$, the traffic becomes quite smooth over time. At $5 \mathrm{~s}$, the variation in traffic density ranges from 0.07 to 0.17 , while at $15 \mathrm{~s}$ this variation is 0.1 to 0.14 , and at $30 \mathrm{~s}$ the range is only 0.12 to 0.13 . Figure 3 presents the normalized traffic density behavior with the proposed model and $b=2 \mathrm{~s}$ at four different time instants. There are larger variations in the density than with $b=1$, but smaller than with the KG model. The traffic evolves into two clusters with a smooth density between them. The variation in density is between 0.09 and 0.16 at $15 \mathrm{~s}$, and between 0.1 and 0 . 15 at $30 \mathrm{~s}$.

The traffic velocity behavior with the KG model is given in Fig. 4 at four different time instants. The greatest fluctuations in velocity occur at $5 \mathrm{~s}$. At $15 \mathrm{~s}$, the traffic has a nearly uniform velocity beyond $50 \mathrm{~m}$ of $31 \mathrm{~m} / \mathrm{s}$.

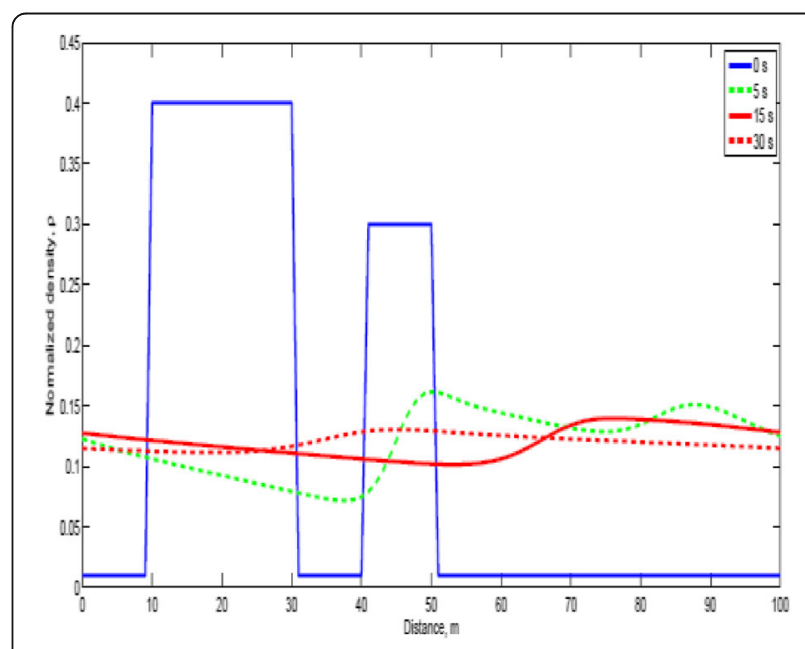

Fig. 2 The proposed model density behavior with $b=1$ 


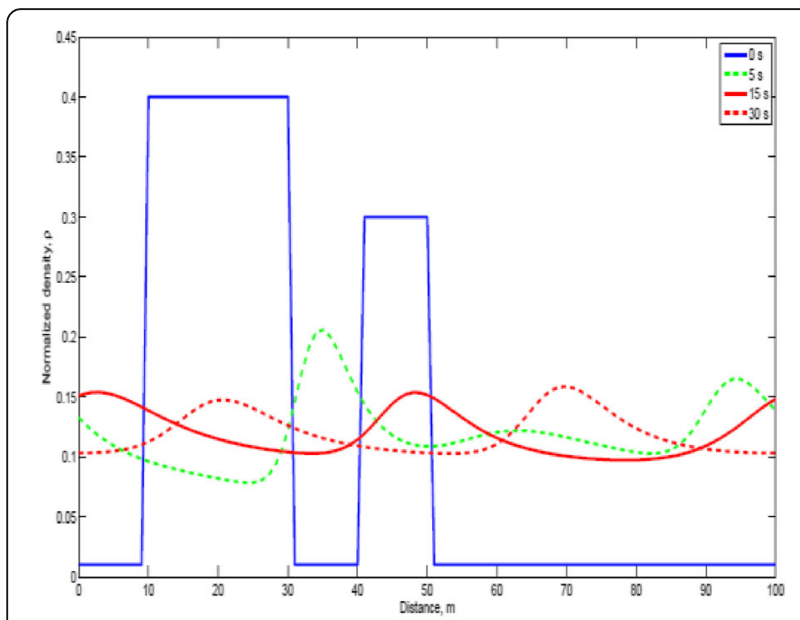

Fig. 3 The proposed model density behavior with $b=2$

There are two clusters of vehicles from 0 to $50 \mathrm{~m}$. The velocity in these clusters varies from $27 \mathrm{~m} / \mathrm{s}$ to $30.2 \mathrm{~m} / \mathrm{s}$. At $30 \mathrm{~s}$, between 0 and $40 \mathrm{~m}$ the traffic has a near uniform velocity of $31 \mathrm{~m} / \mathrm{s}$, and the two clusters are located beyond $40 \mathrm{~m}$. The first cluster is between 40 and $70 \mathrm{~m}$ and has a velocity which varies from 26 to $30.2 \mathrm{~m} / \mathrm{s}$, while the second cluster is located between 70 and $90 \mathrm{~m}$ and has a velocity which varies from 28 to $30.2 \mathrm{~m} / \mathrm{s}$. Comparing the traffic at 15 and $30 \mathrm{~s}$, the velocity in the second cluster increases by $1 \mathrm{~m} / \mathrm{s}$, whereas the velocity of the first cluster decreases by $2 \mathrm{~m} / \mathrm{s}$.

Figure 5 presents the velocity behavior at four different time instants for the proposed model with $b=1$. This corresponds to the density shown in Fig. 2 . At $5 \mathrm{~s}$, the variations in velocity are the greatest, ranging from 29 to $31 \mathrm{~m} / \mathrm{s}$. At $15 \mathrm{~s}$, this variation is 29.5 to $31.5 \mathrm{~m} / \mathrm{s}$, while at $30 \mathrm{~s}$, the difference is less than $1 \mathrm{~m} / \mathrm{s}$. These variations are smaller than with the KG model. Figure 6 presents

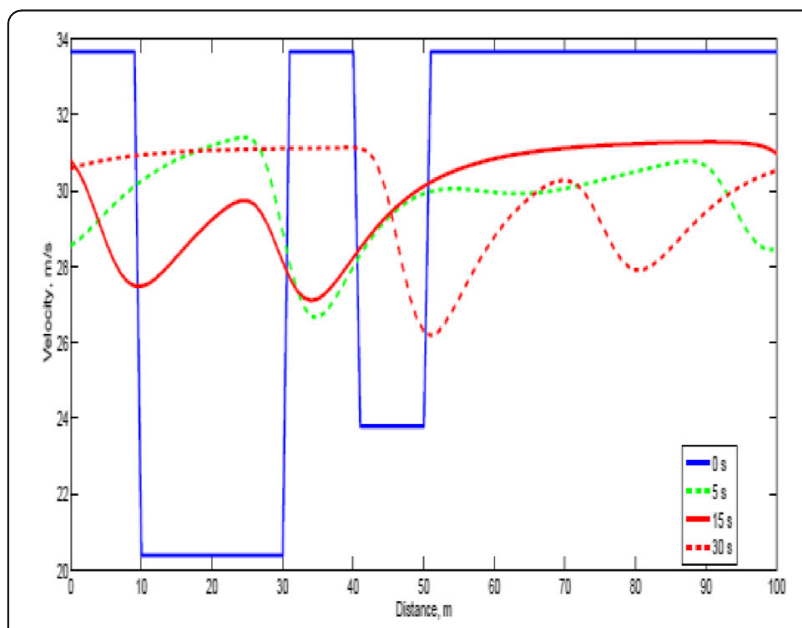

Fig. 4 The KG model velocity behavior with $\tau=1 \mathrm{~s}$

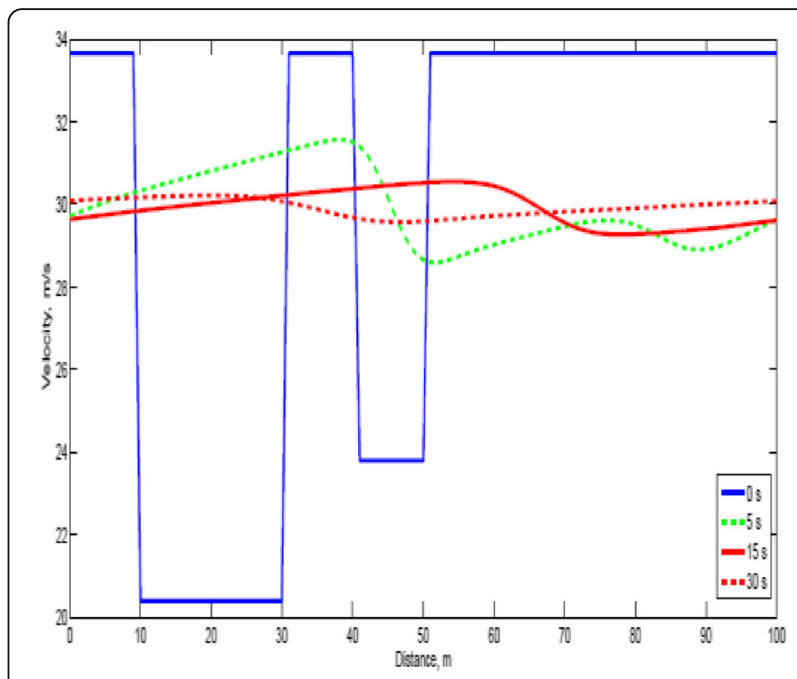

Fig. 5 The proposed model velocity behavior with $b=1$

the velocity behavior at four different time instants for the proposed model with $b=2$. This corresponds to the density shown in Fig. 3. The fluctuations in velocity are greatest at $5 \mathrm{~s}$, with a range of 28 to $31 \mathrm{~m} / \mathrm{s}$, At $15 \mathrm{~s}$, the range is 29 to $30.5 \mathrm{~m} / \mathrm{s}$, while at $30 \mathrm{~s}$ it is only 29 to 30 . $2 \mathrm{~m} / \mathrm{s}$. Thus, the velocity fluctuations are larger than with $b=1$, but smaller than with the KG model.

The traffic flow behavior with the KG model is presented in Fig. 7 at four different time instants. The change in flow follows the changes in density and velocity as it is the product of these two parameters. At $5 \mathrm{~s}$, the flow is more oscillatory, whereas at $15 \mathrm{~s}$ the flow evolves into two clusters between 0 and $50 \mathrm{~m}$. The flow in the first cluster varies from $6 \mathrm{veh} / \mathrm{s}$ to $3.5 \mathrm{veh} / \mathrm{s}$, while in the second cluster it varies from $6.0 \mathrm{veh} / \mathrm{s}$ to $2.8 \mathrm{veh} /$ $\mathrm{s}$. The flow beyond $50 \mathrm{~m}$ aligns to a uniform level of 2.8

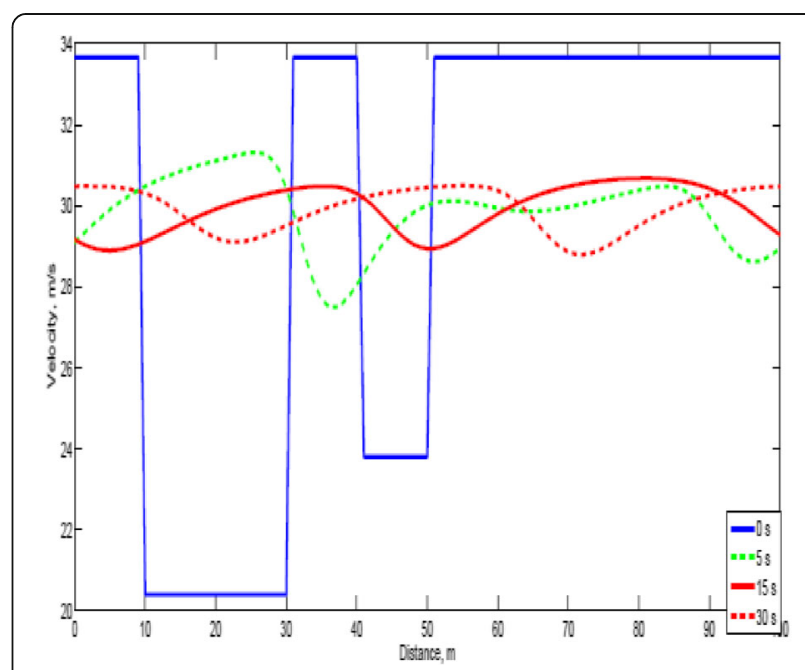

Fig. 6 The proposed model velocity behavior with $b=2$ 


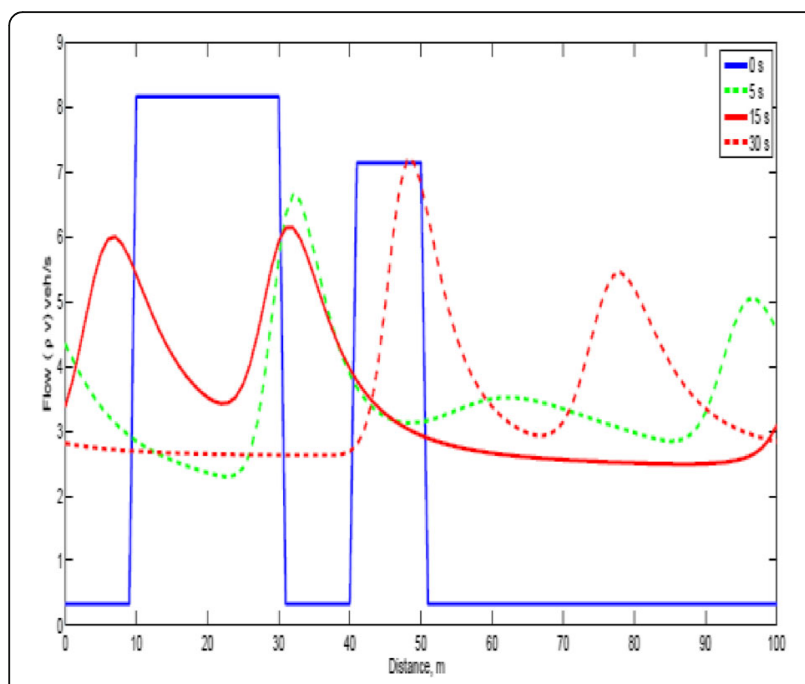

Fig. 7 The $K G$ model flow with $\tau=1 \mathrm{~s}$

veh/s. At $30 \mathrm{~s}$, the two clusters have moved beyond $40 \mathrm{~m}$. The flow in the first cluster now varies from 2.8 to $7.0 \mathrm{veh} / \mathrm{s}$, while in the second cluster it varies from 3 . 0 to $5.2 \mathrm{veh} / \mathrm{s}$. The minimum flow between the clusters is $3.0 \mathrm{veh} / \mathrm{s}$ at $65 \mathrm{~m}$. In the first $40 \mathrm{~m}$, the flow has an approximately uniform level of $2.8 \mathrm{veh} / \mathrm{s}$.

Figure 8 presents the traffic flow behavior at four different time instants with the proposed model and $b=1$. At $5 \mathrm{~s}$, the flow varies from 2.5 to $4.5 \mathrm{veh} / \mathrm{s}$. The maximum and minimum flows occur at $50 \mathrm{~m}$ and $40 \mathrm{~m}$, respectively. At $15 \mathrm{~s}$, the flow varies from 3.2 to $4.2 \mathrm{veh} / \mathrm{s}$, which is less than at $5 \mathrm{~s}$. The maximum and minimum flows now occur at $70 \mathrm{~m}$ and close to $60 \mathrm{~m}$, respectively. At $30 \mathrm{~s}$, the flow is only in the range 3.5 to $4.0 \mathrm{veh} / \mathrm{s}$, and the maximum flow occurs at $40 \mathrm{~m}$. Figure 9 presents the corresponding traffic flow behavior with the

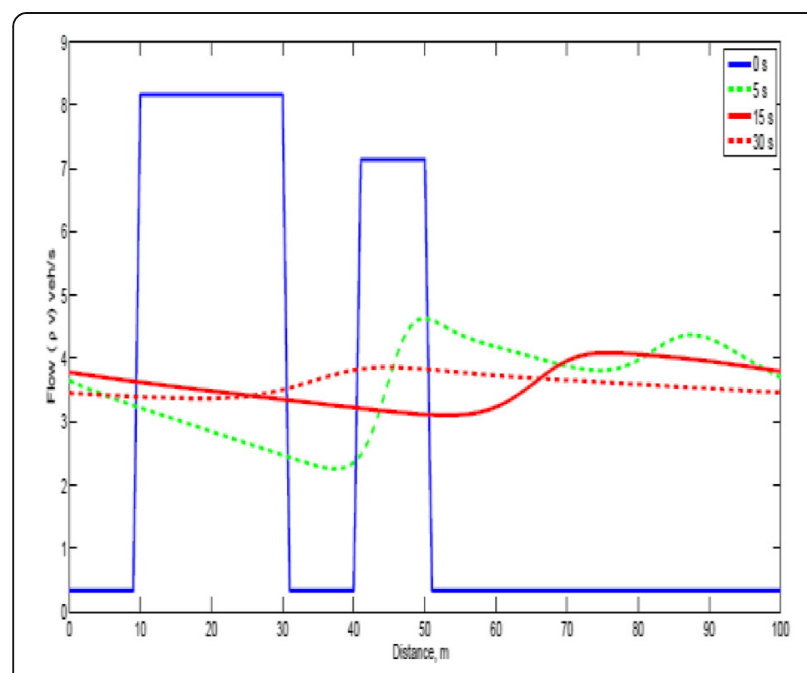

Fig. 8 The proposed model flow with $b=1$

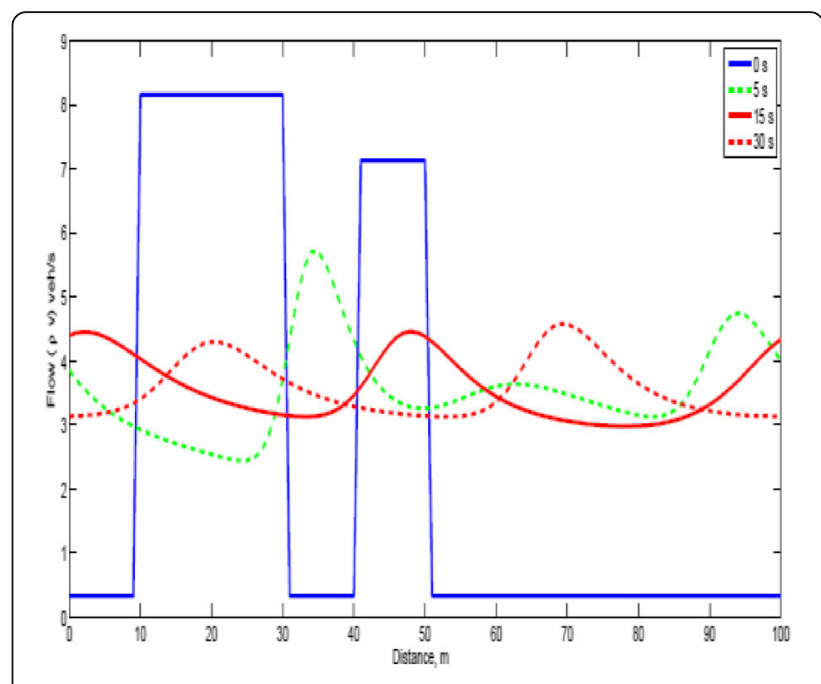

Fig. 9 The proposed model flow with $b=2$

proposed model and $b=2$. The behavior is more oscillatory at $5 \mathrm{~s}$, and the flow varies from 2.5 to $6.0 \mathrm{veh} / \mathrm{s}$, which is greater than with $b=1$. At $15 \mathrm{~s}$, the flow varies from 3.0 to $4.5 \mathrm{veh} / \mathrm{s}$, and it is almost the same at $30 \mathrm{~s}$. However, the locations of the maximum and minimum traffic flows are different.

The velocity behavior on the road over a time span of $3 \mathrm{~s}$ with the PW model is given in Fig. 10. This shows that the variations in velocity increase over time. In particular, the velocity exceeds $300 \mathrm{~m} / \mathrm{s}$ and goes below $200 \mathrm{~m} / \mathrm{s}$, even though the maximum and minimum velocities are $34 \mathrm{~m} / \mathrm{s}$ and $0 \mathrm{~m} / \mathrm{s}$, respectively. Thus the PW model produces unrealistic behavior. Further, the velocity is more oscillatory with the PW model than with the proposed model. The traffic velocity behavior with the Zhang model over a time span of $4 \mathrm{~s}$ is shown in Fig.

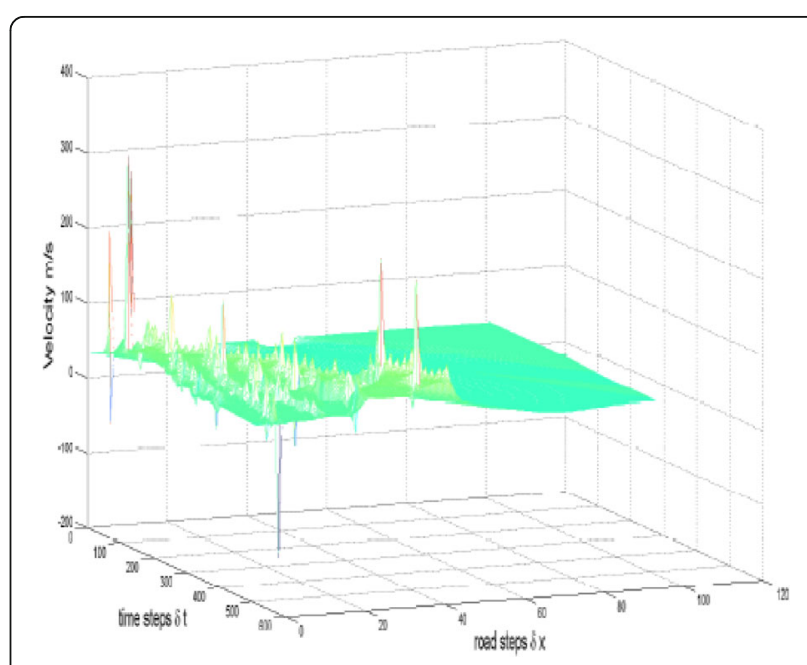

Fig. 10 The PW model velocity behavior 
11. The traffic velocity goes up to $140 \mathrm{~m} / \mathrm{s}$, which is well above the maximum velocity $34 \mathrm{~m} / \mathrm{s}$. Over time, the variations in velocity decrease. The velocity is more oscillatory than the proposed model and is unrealistic. The results in this section show that the flow regulation parameter $b$ in the proposed model can be used to adjust traffic oscillations and cluster behavior. For smaller values of $b$, traffic becomes more uniform. Thus, unrealistic oscillations can be eliminated with this parameter, and traffic behavior can be properly characterized.

\section{Conclusion}

In this paper, a new model was proposed to characterize the physiological and psychological response of drivers to changes in the traffic flow. For a slow response, the traffic becomes clustered, while for a fast response the traffic flow is more uniform. A regulation parameter was introduced to characterize driver response to forward conditions. This allows for more realistic traffic characterization than with other models in the literature. With smaller values of the regulation parameter in the proposed model, changes in velocity are reduced and the traffic flow becomes smooth. Pollution emissions increase with changes in velocity, in particular carbon monoxide, nitric oxide and hydrocarbon emissions are greater at higher velocities. However, velocities between $16.7 \mathrm{~m} / \mathrm{s}$ and 22 . $2 \mathrm{~m} / \mathrm{s}$ result in reduced fuel consumption and

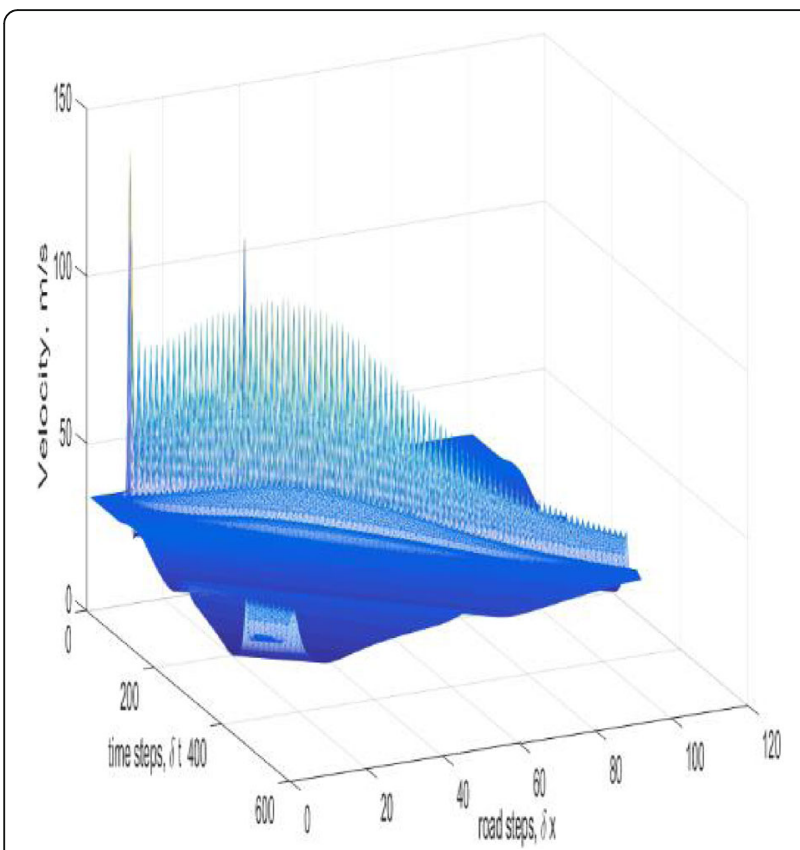

Fig. 11 The Zhang model velocity behavior pollution [32]. Variations in velocity can be reduced with the regulation parameter $b$ in autonomous vehicles to reduce fuel consumption and pollution. The proposed model can help in analyzing the behavior of autonomous vehicles. This will lead to more accurate results which can be employed to reduce fuel consumption and pollution.

\section{Appendix}

A. Eigenvalues $\lambda_{1,2}$

(Eigenvalues)

$$
A(G)=\left(\frac{0}{-v^{2}+\left(\frac{v^{2}(\rho)-v^{2}}{2 d_{t r}}\right)} \frac{1}{2 v}\right) .
$$

At the road segment boundaries, the eigenvalues $\lambda_{i}$ of the Jacobian matrix are required to obtain the flux in (21), and are obtained as the solution of

$$
|A(G)-\lambda I|=\left|\frac{-\lambda}{-v^{2}+\left(\frac{v^{2}(\rho)-v^{2}}{2 d_{t r}}\right)} \frac{1}{2 v-\lambda}\right|=0,
$$

which gives

$$
\lambda^{2}-2 v \lambda+v^{2}-\left(\frac{v^{2}(\rho)-v^{2}}{2 d_{t r}}\right)=0
$$

The eigenvalues are then

$$
\begin{aligned}
\lambda_{1,2} & =\frac{2 v \pm \sqrt{4 v^{2}-4\left(v^{2}-\left(\frac{v^{2}(\rho)-v^{2}}{2 d_{t r}}\right)\right)}}{2} \\
& =v \pm \sqrt{\left(\frac{v^{2}(\rho)-v^{2}}{2 d_{t r}}\right)} .
\end{aligned}
$$

B. Zhang Model Average Velocity

Using Roe scheme, the average velocity at the boundary of segments $i$ and $i+1$ for the Zhang model is

$$
v_{i+\frac{1}{2}}=\frac{-b+\sqrt{b^{2}-4 a c}}{2 a}
$$

where

$$
a=\rho_{i+\frac{1}{2}}-\rho_{i-\frac{1}{2}},
$$




$$
\begin{aligned}
b= & -\left[\rho_{i+\frac{1}{2}} v\left(\rho_{i+\frac{1}{2}}\right)-\rho_{i-\frac{1}{2}} v\left(\rho_{i-\frac{1}{2}}\right)\right]-v(\rho) \\
& \times\left(\rho_{i+\frac{1}{2}}-\rho_{i-\frac{1}{2}}\right)-2 \rho_{i+\frac{1}{2}}\left(v_{i+\frac{1}{2}}-v\left(\rho_{i+\frac{1}{2}}\right)\right) \\
& +2 \rho_{i-\frac{1}{2}}\left(v_{i-\frac{1}{2}}-v\left(\rho_{i-\frac{1}{2}}\right)\right), \\
c= & v(\rho)\left[\rho_{i+\frac{1}{2}} v\left(\rho_{i+\frac{1}{2}}\right)-\rho_{i-\frac{1}{2}} v\left(\rho_{i-\frac{1}{2}}\right)\right]+v(\rho) \\
& \times\left(\rho_{i+\frac{1}{2}}\left(v_{i+\frac{1}{2}}-v\left(\rho_{i+\frac{1}{2}}\right)\right)-\rho_{i-\frac{1}{2}}\left(v_{i-\frac{1}{2}}-v\left(\rho_{i-\frac{1}{2}}\right)\right)\right) \\
& -\left(\frac{\left(\left(v_{i+\frac{1}{2}}-v\left(\rho_{i+\frac{1}{2}}\right)\right) \rho_{i+\frac{1}{2}}\right)^{2}}{\rho_{i+\frac{1}{2}}}+v\left(\rho_{i+\frac{1}{2}}\right)\left(\left(v_{i+\frac{1}{2}}-v\left(\rho_{i+\frac{1}{2}}\right)\right) \rho_{i+\frac{1}{2}}\right)\right) \\
& +\left(\frac{\left(\left(v_{i-\frac{1}{2}}-v\left(\rho_{i-\frac{1}{2}}\right)\right) \rho_{i-\frac{1}{2}}\right)}{\rho_{i-\frac{1}{2}}}+v\left(\rho_{i-\frac{1}{2}}\right)\left(\left(v_{i-\frac{1}{2}}-v\left(\rho_{i-\frac{1}{2}}\right)\right) \rho_{i-\frac{1}{2}}\right)\right) .
\end{aligned}
$$

$$
\begin{aligned}
& e|\Lambda| e^{-1}=\left(\frac{1}{v_{i+\frac{1}{2}}-v\left(\rho_{i+\frac{1}{2}}\right)-\rho_{i+\frac{1}{2}} v\left(\rho_{i+\frac{1}{2}}\right)} \frac{1}{v_{i+\frac{1}{2}}-v\left(\rho_{i+\frac{1}{2}}\right)}\right) \times \\
& \left(\frac{\left|v_{i+\frac{1}{2}}-v\left(\rho_{i+\frac{1}{2}}\right)-\rho_{i+\frac{1}{2}} v\left(\rho_{i+\frac{1}{2}}\right)_{\rho}\right|}{0} \frac{0}{\left|v_{i+\frac{1}{2}}-v\left(\rho_{i+\frac{1}{2}}\right)\right|}\right) \\
& \times\left(\frac{v_{i+\frac{1}{2}}-v\left(\rho_{i+\frac{1}{2}}\right)}{-v_{i+\frac{1}{2}}+v\left(\rho_{i+\frac{1}{2}}\right)+v\left(\rho_{i+\frac{1}{2}}\right)_{\rho}} \frac{-1}{1}\right) \\
& \times \frac{1}{\rho_{i+\frac{1}{2}} v\left(\rho_{i+\frac{1}{2}}\right)} \text {, }
\end{aligned}
$$

\section{Publisher's Note}

\section{Jacobian Matrix for Entropy Fix}

The Jacobian matrix $e|\Lambda| e^{-1}$ for both the proposed and KG models is

$$
\begin{aligned}
& e|\Lambda| e^{-1}=\left(\frac{1}{v_{i+\frac{1}{2}}+\sqrt{\frac{\left|v^{2}\left(\rho_{i+\frac{1}{2}}\right)-v_{i+\frac{1}{2}}^{2}\right|}{2 d_{t r}}}} \frac{1}{v_{i+\frac{1}{2}}-\sqrt{\frac{\left|v^{2}\left(\rho_{i+\frac{1}{2}}\right)-v_{i+\frac{1}{2}}^{2}\right|}{2 d_{t r}}}}\right) \times \\
& \left(\frac{\mid v_{i+\frac{1}{2}}+\sqrt{\frac{\left|v^{2}\left(\rho_{i+\frac{1}{2}}\right)-v_{i+\frac{1}{2}}^{2}\right|}{2 d_{t r}} \mid}}{0} \mid \frac{0}{v_{i+\frac{1}{2}}-\sqrt{\frac{\left|v^{2}\left(\rho_{i+\frac{1}{2}}\right)-v_{i+\frac{1}{2}}^{2}\right|}{2 d_{t r}} \mid}}\right) \\
& \times\left(\frac{v_{i+\frac{1}{2}}-\sqrt{\frac{\left|v^{2}\left(\rho_{i+\frac{1}{2}}\right)-v_{i+\frac{1}{2}}^{2}\right|}{2 d_{t r}}}}{-v_{i+\frac{1}{2}}-\sqrt{\frac{\left|v^{2}\left(\rho_{i+\frac{1}{2}}\right)-v_{i+\frac{1}{2}}^{2}\right|}{2 d_{t r}}}}-1\right) \times
\end{aligned}
$$

$$
\frac{-1}{2 \sqrt{\frac{\left|v^{2}\left(\rho_{i+\frac{1}{2}}\right)-v_{i+\frac{1}{2}}^{2}\right|}{2 d_{t r}}}},
$$

The Jacobian matrix for the PW model is

$$
\begin{aligned}
& e|\Lambda| e^{-1}=\left(\frac{1}{v_{i+\frac{1}{2}}+C_{0}} \frac{1}{v_{i+\frac{1}{2}}-C_{0}}\right) \times \\
& \left(\frac{\left|v_{i+\frac{1}{2}}+C_{0}\right|}{0} \frac{0}{\left|v_{i+\frac{1}{2}}-C_{0}\right|}\right) \times\left(\frac{v_{i+\frac{1}{2}}-C_{0}}{-v_{i+\frac{1}{2}}-C_{0}} \frac{-1}{1}\right) \times \frac{-1}{2 C_{0}},
\end{aligned}
$$

and the Jacobian matrix for the Zhang model is
Springer Nature remains neutral with regard to jurisdictional claims in published maps and institutional affiliations.

\section{Author details}

'Electrical Engineering Department, University of Engineering and Technology, Peshawar, Pakistan. ${ }^{2}$ Department of Electrical and Computer Engineering, University of Victoria, PO Box 1700, STN CSC, Victoria, BC V8W 2Y2, Canada.

Received: 12 September 2017 Accepted: 5 April 2018

Published online: 20 June 2018

\section{References}

1. Davoodi N, Soheili AR, Hashemi SM (2016) A macro-model for traffic flow with consideration of driver's reaction time and distance. Nonlinear Dynam 83:1621-1628

2. Del Castillo JM, Pintado P, Benitez FG (1994) The reaction time of drivers and the stability of traffic flow. Transpn Res B 28:35-60

3. Jamshidnejad A, Papamichail I, Papageorgiou M, Schutter B (2017) A mesoscopic integrated urban traffic flow emission model. Transpn Res C 75:45-83

4. Newell GF (1961) Nonlinear effects in the dynamics of car following. Operations Res 9:209-229

5. Berthaume AL (2015) Microscopic modeling of driver behavior based on modifying field theory for work zone application. Dissertation, University of Massachusetts Amherst

6. Chandler RE, Herman R, Montroll EW (1958) Traffic dynamics: studies in car following. Operations Res 6:165-184

7. Gazis DC, Herman R, Rothery RW (1961) Non-linear follow the leader models of traffic flow. Operations Res 9:545-567

8. Gipps PG (1981) A behavioral car following model for computer simulation. Transpn Res B 15:105-111

9. Wiedemann R (1974) Simulation des straßenverkehrsflusses. Schriftenreihe des IfV, Institut für Verkehrswesen, Universität Karlsruhe

10. Higgs B, Abbas M M, Medina A (2011) Analysis of the Wiedemann car following model over different speeds using naturalistic data. Proc Int Conf on Road Safety and Simulation

11. Romoser MRE, Fisher DL (2009) The effect of active versus passive training strategies on improving older drivers' scanning in intersections. Hum Factors 51:652-668

12. Pollatsek A, Romoser MRE, Fisher DL (2012) Identifying and remediating failures of selective attention in older drivers. Curr Dir Psychol Sci 21:3-7

13. Liu Z, Zhao Y, Wang P (2012) Road traffic safety engineering. Higher Education Press, Beijing

14. Mourant RR, Rockwell TH (1972) Strategies of visual search by novice and experienced drivers. Hum Factors 14:325-335

15. Payne HJ (1971) Models of freeway traffic and control. Simulation Council Proc 1:51-61

16. Whitham GB (1974) Linear and nonlinear waves. Wiley, New York 
17. Daganzo CF (1995) Requiem for second-order fluid approximations of traffic flow. Transpn Res B 29:277-286

18. Balogun SK, Shenge NA, Oladipo SE (2012) Psychosocial factors influencing aggressive driving among commercial and private automobile drivers in Lagos metropolis. Social Sci J 49:83-89

19. Zhang HM (1998) A theory of non-equilibrium traffic flow. Transpn Res B $32: 485-498$

20. Zhang HM (2002) A non-equilibrium traffic model devoid of gas-like behaviour. Transpn Res B 36:275-290

21. Khan Z (2016) Traffic flow modelling for intelligent transportation systems. Dissertation, University of Victoria

22. Emerson JL, Johnson AM, Dawson JD, Uc EY, Anderson SW, Rizzo M (2012) Predictors of driving outcomes in advancing age. Psychol Aging 27:550-559

23. Hindmarch I, Kerr JS, Sherwood N (1991) The effects of alcohol and other drugs on psychomotor performance and cognitive function. Alcohol Alcohol 26:71-79

24. Guo M, Li S, Wang L, Chai M, Chen F, Wei Y (2016) Research on the relationship between reaction ability and mental state for online assessment of driving fatigue. Int J Environ Res Public Health 13:1174-1189

25. Morgan JV (2002) Numerical methods for macroscopic traffic models. Dissertation, University of Reading

26. Greenshields BD (1935) A study in highway capacity. Proc Highway Res Board 14:448-477

27. Roe PL (1981) Approximate Riemann solvers, parameter vectors, and difference schemes. J Comput Phys 43:357-372

28. Leer BV, Thomas JL, Roe PL, Newsome RW (1987) A comparison of numerical flux formulas for the Euler and Navier-stokes equations. Proc American Inst Aeronautics and Astronautics Computational Fluid Dynamics Conf 87-1104:36-41

29. Toro EF (2009) Riemann solvers and numerical methods for fluid dynamics. Springer, Berlin

30. Harten A, Hayman JM (1983) Self adjusting grid methods for onedimensional hyperbolic conservation laws. J Comput Phys 50:253-269

31. LeVeque RJ (1992) Numerical methods for conservation Laws. Springer, Birkhäuser, Basel

32. Nasir MK, Noor RM, Kalam MA, Masum BM (2014) Reduction of fuel consumption and exhaust pollutant using intelligent transport systems. Scientific World J 2014:836375

\section{Submit your manuscript to a SpringerOpen ${ }^{\circ}$ journal and benefit from:}

- Convenient online submission

- Rigorous peer review

- Open access: articles freely available online

- High visibility within the field

- Retaining the copyright to your article

Submit your next manuscript at $\gg$ springeropen.com 